\title{
Kruppel-like factor 2 regulates thymocyte and T-cell migration
}

\author{
Corey M. Carlson ${ }^{1 \star}$, Bart T. Endrizzi ${ }^{1 \star}$, Jinghai Wu ${ }^{2}$, Xiaojie Ding ${ }^{1}$, Michael A. Weinreich ${ }^{1}$, Elizabeth R. Walsh ${ }^{1}$, \\ Maqsood A. Wani ${ }^{2}$, Jerry B. Lingrel ${ }^{2}$, Kristin A. Hogquist ${ }^{1} \&$ Stephen C. Jameson ${ }^{1}$
}

\begin{abstract}
Mammalian Kruppel-like transcription factors are implicated in regulating terminal differentiation of several tissue types ${ }^{1-3}$. Deficiency in Kruppel-like factor (KLF) 2 (also known as LKLF) leads to a massive loss of the peripheral T-cell pool $^{4}$, suggesting KLF2 regulates T-cell quiescence and survival ${ }^{4-7}$. Here we show, however, that KLF2 is essential for T-cell trafficking. KLF2deficient $\left(K l f 2^{-/-}\right)$thymocytes show impaired expression of several receptors required for thymocyte emigration and peripheral trafficking, including the sphingosine-1-phosphate (S1P) receptor $S 1 P_{1}, C D 62 L$ and $\beta_{7}$ integrin. Furthermore, KLF2 both binds and transactivates the promoter for $\mathrm{S}_{1} \mathrm{P}_{1}-$ a receptor that is critical for thymocyte egress and recirculation through peripheral lymphoid organs. Our findings suggest that KLF2 serves to license mature $T$ cells for trafficking from the thymus and recirculation through secondary lymphoid tissues.
\end{abstract}

Kruppel-like factors (KLFs) are a family of zinc-finger transcription factors including at least 15 mammalian family members ${ }^{2}$. KLFs have critical roles in the development of specific cell lineages, as demonstrated by the profound phenotypes that result from genetargeting KLF family members ${ }^{1,2}$. Several KLFs have an important role in cell maturation-a feature exemplified by erythroid KLF (KLF1), which is essential for erythrocyte production of adult-form haemoglobin ${ }^{1-3}$.

KLF2 is expressed in lung, endothelial cells and lymphocytes ${ }^{4,8-11}$ and is essential for normal blood-vessel integrity and lung development ${ }^{4,9-11}$. Although $K l f 2^{-1-}$ thymocyte development is grossly normal, few KLF2-deficient T cells are found in peripheral lymphoid tissues ${ }^{4,6}$. Moreover, the few Klf2 $2^{-l-} \mathrm{T}$ cells that are present in peripheral tissues show signs of activation and induction of cell death, suggesting KLF2 may have a critical role in T-cell quiescence and survival ${ }^{4,6}$. KLF2 is normally expressed in mature thymocytes, naive $\mathrm{T}$ cells and memory $\mathrm{T}$ cells, but its expression is dramatically downregulated with T-cell receptor activation ${ }^{4,12-14}$. Overexpression of KLF2 in the Jurkat T cell line leads to inhibition of cell-cycle progression, an effect that may involve KLF2 repression of $c$-myc transcription and/or induction of $p 21^{\text {WAF1/CIP1 }}$ (refs 6, 15, 16). Taken together, these features have lead to the proposal that KLF2 functions to prevent spontaneous activation and subsequent death of mature $\mathrm{T}$ cells ${ }^{4-7}$.

To test this model, we studied maintenance of $\mathrm{Klf}^{-1-} \mathrm{T}$ cells in vivo. Because murine KLF2 deficiency is embryonic lethal ${ }^{9,11}$, we generated fetal liver chimaeras (FLCs) using embryonic day 12.5 fetal livers from $\mathrm{Klf}^{-1-}$ or $\mathrm{Klf} 2^{+/-}$donors injected into irradiated Rag $2^{-I-}$ hosts (see Methods). After allowing haematopoietic reconstitution, we studied the T-cell pool in the thymus and peripheral lymphoid tissues of the chimaeras. Consistent with previous reports, which involved a distinct targeted allele of KLF2 (ref. 4), we found that thymic development of $K l f 2^{-I-} \mathrm{T}$ cells was grossly normal (Fig. 1a, b), but that there was a massive deficit of peripheral $\mathrm{T}$ cells (Fig. 1a, c). Notably, we also observed an increase in the representation of $\mathrm{Klf}^{-1-}$ mature $\mathrm{CD} 4^{+} \mathrm{CD}^{-}$(CD4 single-positive; CD4 SP) and $\mathrm{CD} 4^{-} \mathrm{CD}^{+}$(CD8 single-positive; $\mathrm{CD} 8 \mathrm{SP}$ ) thymocytes compared with controls (Fig. 1b). Similar results were obtained using FLCs generated in lethally irradiated C57BL/6 (rather than Rag2 ${ }^{-/-}$) hosts (data not shown).

To study the proposed demise of mature $K l f 2^{-l-} \mathrm{T}$ cells, we performed adoptive transfer of $\mathrm{Klf}^{-l-}$ versus $\mathrm{Klf2} 2^{+/-}$thymocytes into congenic hosts and tracked maintenance of the donor population. Unexpectedly, donor Klf2 ${ }^{-1-} \mathrm{CD} 4 \mathrm{SP}$ cells were recovered at similar total numbers compared to $\mathrm{Klf2} 2^{+/-}$CD4 SP cells 14 days following adoptive transfer (Fig. 2a, b), and even at later time points (30 days post-transfer; data not shown) - results that seemed to conflict with the model that KLF2 was required for mature T-cell survival in the periphery. We considered that KLF2 deficiency might deregulate T-cell cytokine dependence, such that the normal survival cytokines were superfluous for Klf2 $2^{-l-}$ T-cell persistence. However,
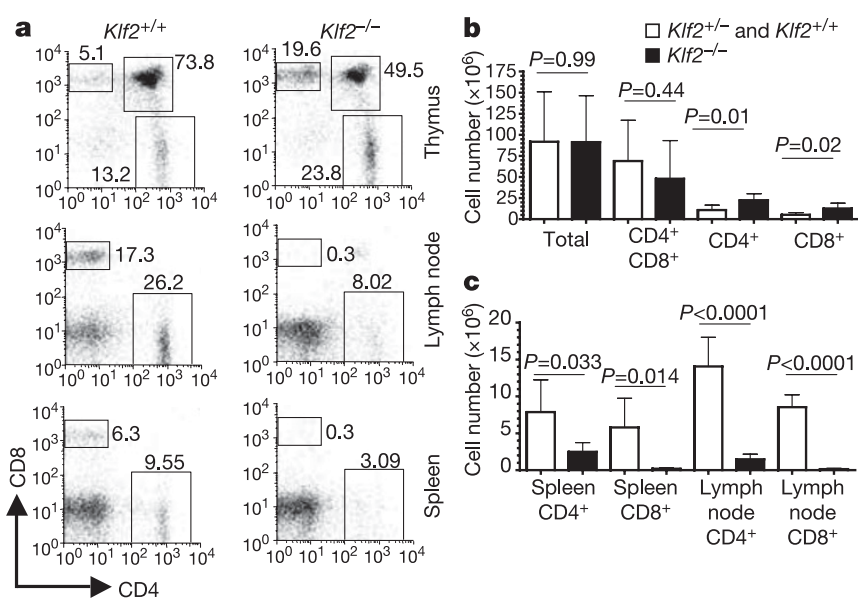

Figure $1 \mid \mathrm{KIf2}^{-/^{-}}$T cells develop but do not populate the periphery in fetal liver chimaeras. Fetal liver chimaeras (FLCs) were generated from $\mathrm{Klf} 2^{-/}$

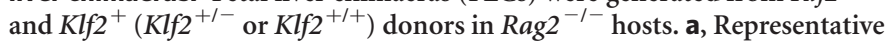
CD4/CD8 staining of the indicated tissues from $\mathrm{Klf2}^{-1-}$ and $\mathrm{Klf2} 2^{+}$FLCs. Values represent the percentage of cells in each boxed region. $\mathbf{b}, \mathbf{c}$, Cell numbers for the indicated populations from thymus (b) and peripheral lymphoid tissues (c). Graphs show the average recovery from multiple experiments ( $n>6$ for $b ; n>4$ for c), with error bars representing standard deviation. Statistical comparisons between $K l f 2^{-I-}$ and $K l f 2^{+}$ groups are indicated. 
in vitro culture of $\mathrm{Klf2}^{-/-}$and $\mathrm{Klf2} 2^{+/-} \mathrm{T}$ cells showed very similar survival characteristics, including dependence on interleukin (IL)-7 for maintenance (Supplementary Fig. S1). As KLF2 deficiency has also been proposed to induce a loss in T-cell quiescence ${ }^{4,6}$, it was possible that $K l f 2^{-1-} \mathrm{T}$ cells underwent spontaneous proliferation in vivo, balancing an increased rate of cell death. To explore this, we labelled proliferating cells in vivo with BrdU (5-bromodeoxyuridine). Both Klf2 ${ }^{-1-}$ and $\mathrm{Klf}^{+/-} \mathrm{T}$ cells showed similar BrdU incorporation following adoptive transfer, arguing against differential proliferation of these populations (Fig. 2c). Although these data suggested $\mathrm{Klf}^{-1-} \mathrm{CD} 4 \mathrm{SP}$ thymocytes were competent for shortterm survival, we noted marked abnormalities in their tissue distribution: Whereas $\mathrm{Klf}^{+/-}$donor T cells were found in blood, lymph nodes and spleen, $\mathrm{Klf}^{-l-}$ T cells segregated almost exclusively to the spleen (Fig. 2a, b), a pattern that was also seen at three and seven days following adoptive transfer (Supplementary Fig. S2a; data not shown). Recovery of both $\mathrm{CD}^{+}$and $\mathrm{CD}^{+}$donor $\mathrm{Klf}^{-/-} \mathrm{T}$ cells was similar to controls soon after adoptive transfer (Supplementary Fig. S2a), but we did observe reduced recovery of $\mathrm{Klf}^{-l-} \mathrm{CD} 8^{+}$(but not $\mathrm{CD}^{+}$) $\mathrm{T}$ cells at later times (days 14 and 30; data not shown). However, it is currently unclear whether KLF2 has a direct role in long-term $\mathrm{CD}^{+}{ }^{+} \mathrm{T}$-cell survival, or if this gradual decline is secondary to altered T-cell trafficking.
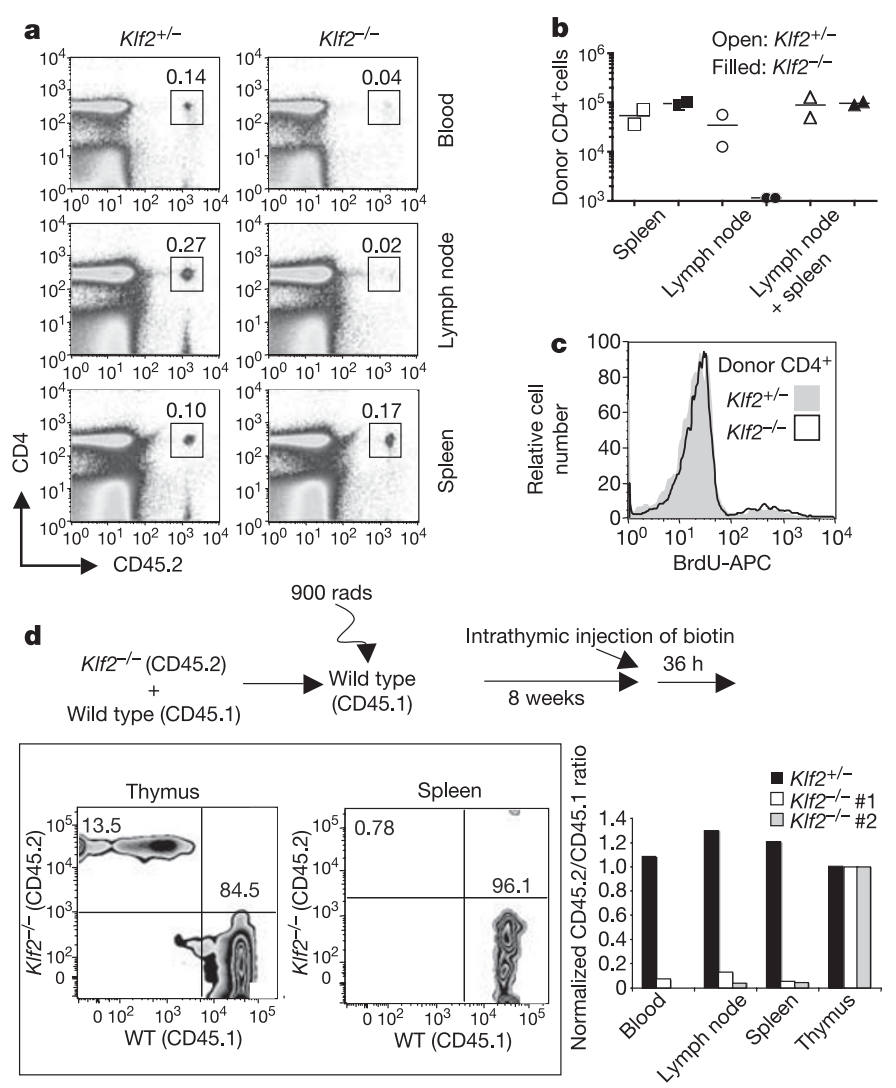

Figure $2 \mid$ KIf $^{-/-}$T cells survive but show deregulated trafficking following adoptive transfer. a, b, Thymocytes from $\mathrm{Klf2}^{+/-}$or $\mathrm{Klf}^{-1-}$ FLCs were transferred into C57BL/6.SJL hosts and labelled with BrdU for 14 days. The percentage (a) and absolute numbers (b) of donor $\mathrm{CD} 4^{+} \mathrm{T}$ cells in the indicated tissues was determined (representative of $n>4$ experiments). c, Representative BrdU incorporation in donor-derived $\mathrm{CD} 4{ }^{+}$splenocytes. An allophycocyanin (APC)-conjugated anti-BrdU antibody was used to detect BrdU in cells. d, The schematic describes the generation and intrathymic injection of mixed bone-marrow chimaeras. Fluorescenceactivated cell sorting (FACS) plots show $\mathrm{Klf2}^{-1-}$ and wild-type biotinylated $\mathrm{CD}^{+}$cells from a representative mixed chimaera. The bar graph indicates the ratio of FLC-derived:normal donor cells in indicated tissues, normalized against the thymic ratio.
These results suggested KLF2 regulates T-cell trafficking, raising the possibility that the absence of $K l f 2^{-/-}$peripheral $\mathrm{T}$ cells might arise from defective thymocyte emigration. To test this model directly, $\mathrm{Klf}^{-1-}$ or $\mathrm{Klf} 2^{+/-}$FLCs were used to generate secondary radiation bone-marrow chimaeras, in which donor FLC cells were placed in competition with congenic wild-type cells (Fig. 2d). Biotin was then administered by intrathymic injection and, $36 \mathrm{~h}$ later, the export of biotin-labelled thymocytes into peripheral lymphoid tissues was determined. Klf2 ${ }^{+/-}$recent thymic emigrants were detected in blood, spleen and lymph nodes in the ratios expected from thymocyte labelling (Fig. 2d). In contrast, $K l f 2^{-I-}$ recent thymic emigrants were very rare, despite efficient labelling of these cells in the thymus (Fig. 2d). These data contrast with those expected if KLF2 deficiency caused T-cell death following thymic egress, in which case most peripheral Klf $2^{-1-} \mathrm{T}$ cells would presumably be thymic emigrants. Hence, these results argue for impaired thymic emigration of $\mathrm{Klf}^{-1-} \mathrm{T}$ cells.

To understand the basis for these altered trafficking patterns, we analysed the phenotype of $K l f 2^{-l-} \mathrm{T}$ cells. $\mathrm{CD} 4^{+} \mathrm{CD} 8^{+}$(doublepositive; DP) thymocytes from $K l f 2^{-/-}$and $K l f 2^{+/-}$chimaeras had very similar phenotypes (Fig. 3a), consistent with the fact that KLF2 is upregulated only after positive selection in the thymus ${ }^{4,17}$. Singlepositive (SP) thymocytes from $K l f 2^{-I-}$ and $K l f 2^{+l-}$ chimaeras showed similar expression of some markers, including $\mathrm{T}$-cell receptor $\beta$ (TCR $\beta$ ), CD25 and CD5 (Fig. 3a; data not shown), but other molecules were altered in KLF2-deficient cells. Klf2 ${ }^{-/-}$SP thymocytes were CD69 $9^{\text {high }}$ and CD62L Low (Fig. 3a), suggestive of a 'semi-mature' phenotype ${ }^{18-20}$; yet these cells were also CD24 ${ }^{\text {low }}$ and Qa2 ${ }^{\text {high }}$ (Fig. 3a; data not shown), a phenotype consistent with full maturity ${ }^{18-20}$. Despite their CD69 expression, the CD24 ${ }^{\text {low }} \mathrm{CD} 25^{\text {low }}$ phenotype of Klf $2^{-I-} \mathrm{T}$ cells argues against them being activated. Furthermore, expression levels of the activation/memory marker CD44 were similar on $\mathrm{Klf}^{-/-}$and $\mathrm{Klf2} 2^{+/-}$thymocytes, although there was considerable variability in CD44 expression in individual FLCs (data not shown). With regard to trafficking, the expression of CD62L, CCR7 and $\beta_{7}$ integrin - all of which are involved in the entry of T cells into peripheral lymphoid tissues ${ }^{21-24}$-was decreased on $\mathrm{Klf}^{-1-}$ $\mathrm{T}$ cells (Fig. 3a). Furthermore, CD69 expression by Klf2 ${ }^{-1-}$ SP thymocytes is potentially relevant, as CD69 can impair T-cell migration ${ }^{19,25,26}$. The rare peripheral $\mathrm{Klf}^{-l-} \mathrm{T}$ cells were phenotypically similar to SP thymocytes (Fig. 3a; data not shown), and the CD $69^{\text {hi }} \mathrm{CD} 2 \mathrm{~L}^{\text {lo }}$ phenotype of Klf2 ${ }^{-1-}$ SP thymocytes was maintained at 6 (Supplementary Fig. S2b), 14 or 30 (data not shown) days after adoptive transfer, indicating that the deregulated expression of these markers by Klf2 $2^{-1-} \mathrm{T}$ cells is stable.

In order to further explore the maturation state and trafficking potential of $\mathrm{Klf}^{-1-}$ SP thymocytes, we sorted $\mathrm{Klf2} 2^{-1-}$ and $\mathrm{Klf2} 2^{+/-}$ DP and CD4 SP thymocytes and performed real-time polymerase chain reaction following reverse transcription (RT-PCR) on genes known to change in expression during thymocyte maturation (Supplementary Fig. S3). Most genes showed a similar pattern of expression in Klf2 $2^{+/-}$and Klf2 $2^{-1-}$ thymocyte populations, consistent with normal thymocyte maturation in the absence of KLF2 (Supplementary Fig. S3). Real-time RT-PCR analysis did, however, demonstrate a dramatic decrease in the expression of mRNA encoding CD62L in $\mathrm{Klf}^{-1-}$ SP thymocytes (Fig. 3b and Supplementary Fig. S3) and a more modest impairment in the expression of $\beta_{7}$ integrin mRNA (Fig. 3b and Supplementary Fig. S3). Notably, loss of KLF2 had no substantial effect on the expression of CCR7 mRNA, although expression of the CCR7 protein was reproducibly reduced on $\mathrm{Klf}^{-l-}$ SP thymocytes (Fig. 3a). Also of note, two molecules involved in the regulation of apoptosis- $\mathrm{Bcl} 2$ and Ian $1-$ were unaffected at the mRNA level by KLF2 loss (Fig. 3b and Supplementary Fig. S3). Although these assays focused on CD4 SP thymocytes, preliminary data indicate similar gene expression patterns in $\mathrm{Klf}^{-/-}$ CD8 SP cells (data not shown).

Reduced expression of CD62L, CCR7 and $\beta_{7}$ integrin immediately 


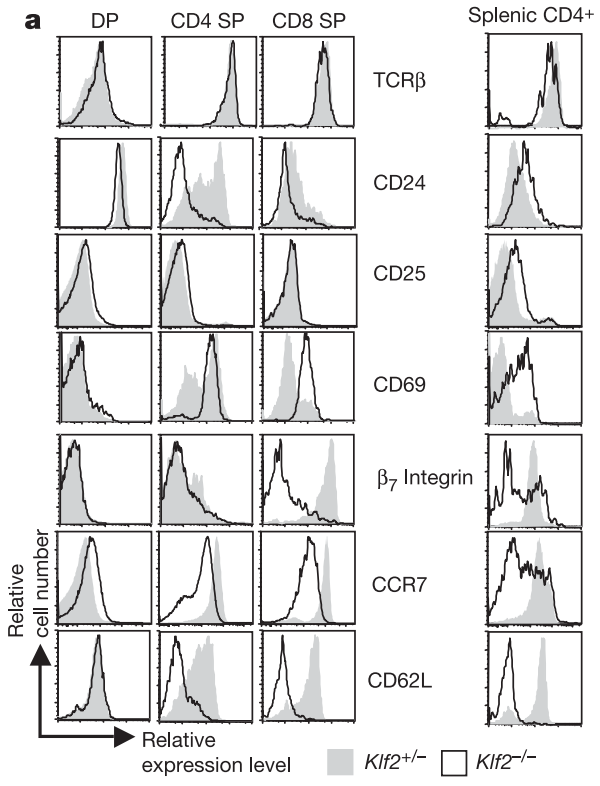

Figure 3 | KLF2 is required for thymocyte expression of critical trafficking molecules. a, Phenotype of thymocytes and $\mathrm{CD}^{+}$splenocytes from $K l f 2^{-l-}$ and $K l f 2^{+/-}$FLCs (representative of $n>4$ experiments). b, S1P CD62L, $\beta_{7}$ integrin and BCL2 mRNA abundance was assessed by real-time b
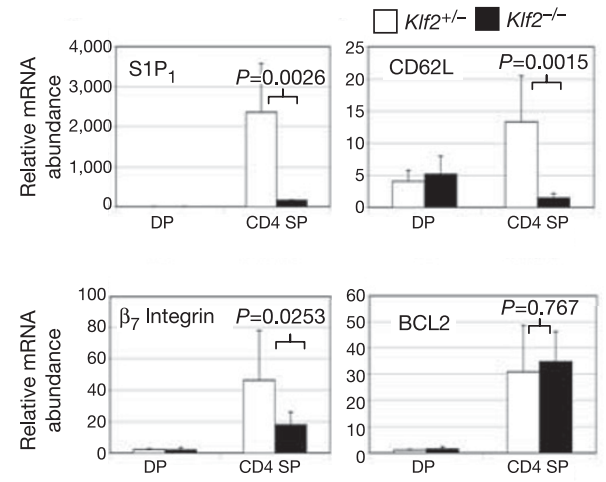

RT-PCR analysis of sorted DP and CD4 SP thymocytes from $\mathrm{Klf}^{-1-}$ and $K l f 2^{+/-}$FLCs. Bar graphs show the average signal from $n \geqq 3$ experiments, with error bars representing standard deviation. $P$-values from comparisons between $\mathrm{Klf2}^{-I-}$ and $\mathrm{Klf2} 2^{+-} \mathrm{CD} 4 \mathrm{SP}$ samples are indicated.

In summary, our data suggest a radically different model of the role of KLF2: rather than primarily influencing T-cell survival, KLF2 regulates thymocyte and T-cell trafficking. Loss of KLF2 results in defective expression of $\mathrm{S}_{1} \mathrm{P}_{1}, \mathrm{CD} 62 \mathrm{~L}, \beta_{7}$ integrin and CCR7, and KLF2 seems to have a direct role in induction of $S 1 P_{1}$ expression. KLF2 and $\mathrm{S}_{1} \mathrm{P}_{1}$ show similar expression patterns in T cells, both being upregulated on thymocyte maturation, downregulated after T-cell activation and re-expressed in the late effector/memory pool ${ }^{4,12,13,27}$. Furthermore, KLF2-null and $\mathrm{S}_{1} \mathrm{P}_{1}$-null mice die at similar stages in gestation because of widespread haemorrhaging, probably owing to defective tunica media integrity ${ }^{9,11,27,30}$. It is interesting to speculate that KLF2 may be required for promoting $\mathrm{S}_{1} \mathrm{P}_{1}$ expression in
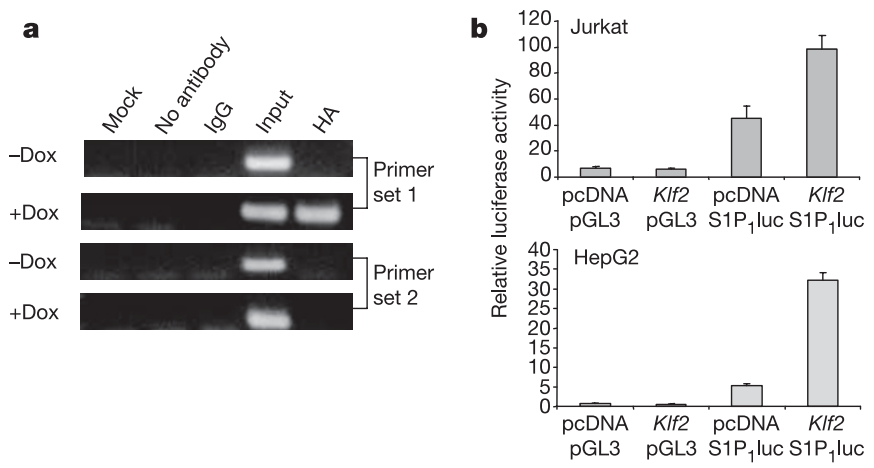

Figure 4 | KLF2 transactivates the $\mathbf{S 1 P}_{\mathbf{1}}$ promoter. a, Jurkat $\mathrm{T}$ cells were induced ("+Dox") or not (“-Dox") to express HA-tagged KLF2 and chromatin immunoprecipitation (ChIP) performed. PCR for $\mathrm{S}_{1} \mathrm{P}_{1}$ promoter fragments was conducted on chromosomal DNA ("Input"), DNA co-precipitated with KLF2 ("HA"), and control immunoprecipitations, as indicated. Primer set 1 amplifies a region immediately upstream of the $\mathrm{S}_{1} \mathrm{P}_{1}$ transcriptional start site (Supplementary Fig. S4), whereas primer set 2 amplifies a region about $300 \mathrm{bp}$ further upstream. b, KLF2 transactivation of the $\mathrm{S}_{1} \mathrm{P}_{1}$ promoter was assessed by reporter assay ${ }^{14,15}$. Jurkat $(\mathrm{T})$ and HepG2 (liver) cells were co-transfected with either Klf2 cDNA ("Klf2") or empty vector ("pcDNA"), plus either the $\mathrm{S}_{1} \mathrm{P}_{1}$ promoter luciferase reporter ("S1 $\mathrm{P}_{1}$ luc") or control plasmid ("pGL3"). Data are representative of $n=3$ experiments. Error bars represent s.d. 
endothelial cells, explaining the early embryonic lethality of KLF2-deficient mice.

Our data do not exclude additional roles for KLF2, potentially including long-term effects on T-cell quiescence or survival. Indeed, forced KLF2 expression can clearly promote cell-cycle withdrawal in tumour cell lines ${ }^{6,15,16}$. However, our findings suggest KLF2 is critical to license mature thymocytes for trafficking competence. Such a role is consistent with the action of other KLF family members, which are essential for terminal differentiation of various cell types ${ }^{1-3}$.

\section{METHODS}

A detailed description of all materials and methods can be found in Supplementary Information.

Mice, chimaeras and adoptive transfer. Fetal livers from embryonic day 12.5 $\mathrm{Klf} 2^{-1-}$ and control animals were used to reconstitute irradiated Rag $2^{-1-}$ hosts, which were analysed after eight weeks. In some experiments, thymocytes from FLCs were adoptively transferred into C57BL/6.SJL hosts, which were then offered BrdU-laced drinking water. Donor-derived $\left(\mathrm{CD} 45.2^{+}\right)$cells were analysed for tissue distribution, phenotype and BrdU incorporation by flow cytometry, using the indicated antibodies (BD Pharmingen). Secondary bonemarrow chimaeras were generated using a mixture of bone marrow from FLCs (CD45.2) plus C57BL/6.SJL (CD45.1) animals to reconstitute lethally irradiated C57BL/6.SJL hosts. After eight weeks, these animals were intrathymically injected with biotin and the appearance of FLC-donor-derived (CD45.2) and wild-type-donor-derived (CD45.1) biotin-labelled cells in the thymus and periphery monitored.

Real-time RT-PCR. Thymocyte subsets were purified by fluorescence-activated cell sorting (FACS) on a FACSVantage (Becton Dickinson), followed by RNA isolation and preparation of cDNA. Real-time RT-PCR was performed on a SmartCycler (Cepheid) using primers listed in Supplementary Information. mRNA abundance was determined relative to controls (Hprt, Gapdh and/or Ctnnb1).

Chromatin immunoprecipitation and gene reporter assays. Jurkat $\mathrm{T}$ cells transfected with tetracycline (tet)-inducible haemagglutinin (HA)-tagged KLF2 (refs 14,15$)$ were cultured with or without doxycycline for $48 \mathrm{~h}$, formaldehydefixed, lysed, and subjected to immunoprecipitation with an anti-HA antibody or control IgG. Co-precipitated DNA was used as template for PCR of the $\mathrm{S}_{1} \mathrm{P}_{1}$ promoter using primer sets 1 or 2 (initiating 141 base pairs (bp) and $474 \mathrm{bp}$ upstream of the indicated $\mathrm{S}_{1} \mathrm{P}_{1}$ transcriptional start site, respectively). For $\mathrm{S}_{1} \mathrm{P}_{1}$ reporter assays, normal Jurkat or HepG2 cells were transiently transfected with the indicated plasmids and luciferase activity monitored at $48 \mathrm{~h}$ using a Monolight 3010 Luminometer (BD Biosciences).

Statistical analysis. An unpaired two-tailed Student's $t$-test was applied using GraphPad software (Prism) on normal or $\log _{10}$-transformed data sets.

\section{Received 18 December 2005; accepted 3 May 2006.}

1. Dang, D. T., Pevsner, J. \& Yang, V. W. The biology of the mammalian Kruppellike family of transcription factors. Int. J. Biochem. Cell Biol. 32, 1103-1121 (2000).

2. Kaczynski, J., Cook, T. \& Urrutia, R. Sp1- and Kruppel-like transcription factors. Genome Biol. 4, 206 (2003)

3. Turner, J. \& Crossley, M. Mammalian Kruppel-like transcription factors: more than just a pretty finger. Trends Biochem. Sci. 24, 236-240 (1999).

4. Kuo, C. T., Veselits, M. L. \& Leiden, J. M. LKLF: a transcriptional regulator of single-positive T cell quiescence and survival. Science 277, 1986-1990 (1997).

5. Kuo, C. T. \& Leiden, J. M. Transcriptional regulation of T lymphocyte development and function. Annu. Rev. Immunol. 17, 149-187 (1999).

6. Buckley, A. F., Kuo, C. T. \& Leiden, J. M. Transcription factor LKLF is sufficient to program T cell quiescence via a c-Myc-dependent pathway. Nature Immunol. 2, 698-704 (2001)

7. Di Santo, J. P. Lung Kruppel-like factor: a quintessential player in T cell quiescence. Nature Immunol. 2, 667-668 (2001).

8. Anderson, K. P., Kern, C. B., Crable, S. C. \& Lingrel, J. B. Isolation of a gene encoding a functional zinc finger protein homologous to erythroid Kruppel-like factor: identification of a new multigene family. Mol. Cell. Biol. 15, 5957-5965 (1995)
9. Wani, M. A., Means, R. T. Jr \& Lingrel, J. B. Loss of LKLF function results in embryonic lethality in mice. Transgenic Res. 7, 229-238 (1998).

10. Wani, M. A., Wert, S. E. \& Lingrel, J. B. Lung Kruppel-like factor, a zinc finger transcription factor, is essential for normal lung development. J. Biol. Chem. 274, 21180-21185 (1999).

11. Kuo, C. T. et al. The LKLF transcription factor is required for normal tunica media formation and blood vessel stabilization during murine embryogenesis. Genes Dev. 11, 2996-3006 (1997).

12. Schober, S. L. et al. Expression of the transcription factor lung Kruppel-like factor is regulated by cytokines and correlates with survival of memory T cells in vitro and in vivo. J. Immunol. 163, 3662-3667 (1999).

13. Grayson, J. M., Murali-Krishna, K., Altman, J. D. \& Ahmed, R. Gene expression in antigen-specific $\mathrm{CD} 8^{+} \mathrm{T}$ cells during viral infection. J. Immunol. 166, 795-799 (2001).

14. $\mathrm{Wu}$, J. \& Lingrel, J. B. Kruppel-like factor 2, a novel immediate-early transcriptional factor, regulates IL-2 expression in T lymphocyte activation. J. Immunol. 175, 3060-3066 (2005)

15. Wu, J. \& Lingrel, J. B. KLF2 inhibits Jurkat T leukemia cell growth via upregulation of cyclin-dependent kinase inhibitor p21 ${ }^{\mathrm{WAF} 1 / \mathrm{CIP} 1}$. Oncogene 23 , 8088-8096 (2004).

16. Haaland, R. E., Yu, W. \& Rice, A. P. Identification of LKLF-regulated genes in quiescent CD4+ T lymphocytes. Mol. Immunol. 42, 627-641 (2005)

17. Mick, V. E., Starr, T. K., McCaughtry, T. M., McNeil, L. K. \& Hogquist, K. A. The regulated expression of a diverse set of genes during thymocyte positive selection in vivo. J. Immunol. 173, 5434-5444 (2004)

18. Gabor, M. J., Godfrey, D. I. \& Scollay, R. Recent thymic emigrants are distinct from most medullary thymocytes. Eur. J. Immunol. 27, 2010-2015 (1997).

19. Feng, C. et al. A potential role for CD69 in thymocyte emigration. Int. Immunol. 14, 535-544 (2002).

20. Boursalian, T. E., Golob, J., Soper, D. M., Cooper, C. J. \& Fink, P. J. Continued maturation of thymic emigrants in the periphery. Nature Immunol. 5, 418-425 (2004)

21. Arbones, M. L. et al. Lymphocyte homing and leukocyte rolling and migration are impaired in L-selectin-deficient mice. Immunity 1, 247-260 (1994).

22. Wagner, N. et al. Critical role for $\beta 7$ integrins in formation of the gutassociated lymphoid tissue. Nature 382, 366-370 (1996).

23. Wagner, N. et al. L-selectin and $\beta 7$ integrin synergistically mediate lymphocyte migration to mesenteric lymph nodes. Eur. J. Immunol. 28, 3832-3839 (1998).

24. Cyster, J. G. Chemokines, sphingosine-1-phosphate, and cell migration in secondary lymphoid organs. Annu. Rev. Immunol. 23, 127-159 (2004).

25. Nakayama, T. et al. The generation of mature, single-positive thymocytes in vivo is dysregulated by CD69 blockade or overexpression. J. Immunol. 168, 87-94 (2002)

26. Shiow, L. R. et al. CD69 acts downstream of interferon- $\alpha / \beta$ to inhibit $S 1 P_{1}$ and lymphocyte egress from lymphoid organs. Nature 440, 540-544 (2006).

27. Matloubian, M. et al. Lymphocyte egress from thymus and peripheral lymphoid organs is dependent on S1P receptor 1. Nature 427, 355-360 (2004).

28. Allende, M. L. Dreier, J. L Mandala, S. \& Proia, R. L. Expression of the sphingosine-1-phosphate receptor, $\mathrm{SPP}_{1}$, on T-cells controls thymic emigration. J. Biol. Chem. 279, 15396-15401 (2004).

29. Lo, C. G., Xu, Y., Proia, R. L. \& Cyster, J. G. Cyclical modulation of sphingosine1-phosphate receptor 1 surface expression during lymphocyte recirculation and relationship to lymphoid organ transit. J. Exp. Med. 201, 291-301 (2005).

30. Liu, Y. et al. Edg-1, the G protein-coupled receptor for sphingosine-1-phosphate, is essential for vascular maturation. J. Clin. Invest. 106, 951-961 (2000).

Supplementary Information is linked to the online version of the paper at www.nature.com/nature.

Acknowledgements We thank J. Cyster for generous provision of CCL19-Fc fusion protein, $\mathrm{Y}$. Li for developing S1P ${ }_{1}$ RT-PCR assays, M. Jenkins and $M$. Mescher for critical input on the manuscript, and members of the 'Jamequist' laboratory for helpful discussions. This work was supported by the NIH (an immunology pre-doctoral training grant to B.T.E., a grant to K.A.H., and a grant to S.C.J.), the American Cancer Society (a grant to S.C.J.), and the Cancer Research Institute (post-doctoral fellowship to C.M.C.).

Author Information Reprints and permissions information is available at npg.nature.com/reprintsandpermissions. The authors declare no competing financial interests. Correspondence and requests for materials should be addressed to S.C.J. (james024@umn.edu) or K.A.H. (hogqu001@umn.edu). 